\title{
Insights into Effective RNAi Gained from Large-Scale siRNA Validation Screening
}

\author{
UTE KRUEGER, TOBIAS BERGAUER, BRIGITTE KAUFMANN, IRENE WOLTER, \\ SABRINA PILK, MIKE HEIDER-FABIAN, STEFFI KIRCH, CHRISTIANE ARTZ-OPPITZ, \\ MEIKE ISSELHORST, and JULIANE KONRAD
}

\begin{abstract}
Transfection of chemically synthesized short interfering RNAs (siRNAs) enables a high level of sequence-specific gene silencing. Although siRNA design algorithms have been improved in recent years, it is still necessary to prove the functionality of a given siRNA experimentally. We have functionally tested several thousand siRNAs for target genes from various gene families including kinases, phosphatases, and cancer-related genes (e.g., genes involved in apoptosis and the cell cycle). Some targets were difficult to silence above a threshold of $70 \%$ knockdown. By working with one design algorithm and a standardized validation procedure, we discovered that the level of silencing achieved was not exclusively dependent on the siRNA sequences. Here we present data showing that neither the gene expression level nor the cellular environment has a direct impact on the knockdown which can be achieved for a given target. Modifications of the experimental setting have been investigated with the aim of improving knockdown efficiencies for siRNA-target combinations that show only moderate knockdown. Use of higher siRNA concentrations did not change the overall performance of the siRNA-target combinations analyzed. Optimal knockdown at the mRNA level was usually reached 48-72 hours after transfection. Target gene-specific characteristics such as the accessibility of the corresponding target sequences to the RNAi machinery appear to have a significant influence on the knockdown observed, making certain targets easy or difficult to knock down using SiRNA.
\end{abstract}

\section{INTRODUCTION}

$\mathbf{R}$ NA INTERFERENCE (RNAi) is a conserved mechanism which has been observed in different species such as fungi, worms, Drosophila, plants, and mammals (Elbashir et al., 2001a; Fire et al., 1998; Hamilton and Baulcombe, 1999; Hammond et al., 2000; Tuschl et al., 1999; Zamore et al., 2000). RNAi is mediated by siRNAs, which are 19-22 nucleotide long, double-stranded RNA molecules with two to three nucleotide overhangs. The silencing machinery is dependent on two multiprotein complexes: (1) DICER, which is responsible for processing of siRNAs from double-stranded RNA mole- cules, and (2) RISC, which after incorporation of the siRNA leads to sequence-specific cleavage of the target mRNA and thus to knockdown of the corresponding gene (reviewed in Aronin, 2006; Collins and Cheng, 2005; Dykxhoorn et al., 2003; Filipowicz, 2005; Hammond, 2005; Hannon, 2002; Zamore and Haley, 2005). Experimental use of RNAi in mammalian cells has been hampered by the observation that long double-stranded RNA molecules lead to nonspecific knockdown via induction of the interferon pathway. Reports by the group of Tuschl (Elbashir et al., 2001b) as well as Caplen et al. (2001) showed that the use of short double-stranded RNA molecules (siRNAs) less than 30 nucleotides cir-

QIAGEN GmbH, Hilden, Germany. 
cumvent this interferon response and instead lead to efficient as well as specific downregulation of genes. RNAi has since become a widely used experimental tool, facilitating loss of function analysis in mammalian cells and target identification in drug screening projects.

Synthetic siRNA molecules are widely used for RNAi experiments, and the silencing capacity of synthetic siRNAs is one critical factor that determines the success of silencing experiments. Some fundamental empirical guidelines for designing efficient siRNA molecules were initially published by the group of Tuschl (Elbashir et al., 2001a; Tuschl, 2002). Further investigations into the RNAi mechanism itself as well as characteristics of siRNAs with either high or low silencing capacity (Holen et al., 2002; Jagla et al., 2005; Khvorova et al., 2003; Miyagishi and Taira, 2005; Reynolds et al., 2004; Schwarz et al., 2003; Ui-Tei et al., 2004) have led to rational and advanced design algorithms which allow selection of potent siRNAs. Although design algorithms have been improved over the last few years, there is still a risk that not all siRNAs chosen will result in significant knockdown within the given experimental setting. Therefore, performance of each siRNA must still be proven experimentally when selecting the most efficient siRNA for loss-of-function studies.

BIOPREDsi developed by Huesken et al. (2005) is one example of an up-to-date design algorithm that efficiently selects potent siRNA sequences. We have experimentally validated several thousand siRNAs covering a variety of gene families using the BIOPREDsi algorithm for siRNA design and a standardized experimental procedure. These functionally tested siRNAs, together with accompanying data generated during the validation process, serve as a useful resource for future loss-of-function studies. We have used the validation data for further indepth analysis on siRNA performance.

During the screening of siRNAs, it became evident that some target genes were difficult to silence $\geq 70 \%$. In an attempt to improve the silencing levels for these genes, the kinetics of knockdown were analyzed, and increased siRNA concentrations were tested. We investigated whether the gene expression level of a given gene or the localization of the siRNA target sequence on the mRNA were any indication as to whether knockdown would be successful. Different siRNA-target combinations were tested in various cell lines to see whether the silencing level was affected by the cellular environment.

\section{MATERIALS AND METHODS}

\section{Cell culture}

HeLa (Cat.-No. ACC 57), HeLa S3 (ACC 161), A549 (ACC 107), HEK293 (ACC 305), and HepG2 (ACC 180) cells were obtained from DSMZ. MCF-7 cells were or- dered from ATCC (No. HTB-22). All cell lines were cultured at $37^{\circ} \mathrm{C}$ in a humidified atmosphere with $5 \% \mathrm{CO}_{2}$. HeLa, A549, and HEK293 cells were grown in DMEM containing 10\% FCS and nonessential amino acids. HepG2 cells were grown in RPMI 1640 containing $10 \%$ FCS and $2 \mathrm{mM}$ glutamine. MCF-7 cells were grown in RPMI 1640 with $10 \%$ FCS, 2 mM glutamine, nonessential amino acids, $1 \mathrm{mM}$ sodium pyruvate, and $10 \mu \mathrm{g} / \mathrm{mL}$ insulin. All cell culture media were supplemented with penicillin $(100 \mathrm{U} / \mathrm{mL})$ and streptomycin $(0.1 \mathrm{mg} / \mathrm{mL})$.

\section{siRNA transfection}

All the synthetic siRNAs were designed at Qiagen using the BIOPREDsi algorithm licensed from Novartis. All siRNA duplexes for which data are shown are listed in the Supplementary Table 1. Information about additional validated siRNAs can be found on the Web at www.qiagen.com/GeneGlobe.

siRNA transfections were performed in 96-well plates. Transfection parameters were optimized for each cell line prior to validation according to the instructions given in the HiPerFect Transfection Reagent handbook. Optimized parameters were $5 \mathrm{nM}$ siRNA in combination with $0.75 \mu \mathrm{L}$ HiPerFect (Qiagen) for HeLa, A549, and HeLa S3 cells or $25 \mathrm{nM}$ siRNA and $1.5 \mu \mathrm{L}$ HiPerFect for HepG2, HEK293, and MCF-7 cells. Usually $2 \times 10^{4}$ cells (for HEK293, the cell number was $4 \times 10^{4}$ ) were plated 24 hours prior to transfection. Briefly, for duplicate transfections, siRNA and HiPerFect were diluted in $100 \mu \mathrm{L}$ DMEM (Gibco, Grand Island, NY) without serum and incubated for $10 \mathrm{~min}$ at room temperature. After cell culture medium removal, $100 \mu \mathrm{L}$ fresh medium and $50 \mu \mathrm{L}$ transfection complexes were added per well. HepG2 cells were transfected according to the reverse transfection protocol described in the HiPerFect handbook. Following this protocol, $4 \times 10^{4}$ cells were plated simultaneously with transfection complexes. Cells were incubated for 48 hours before analyzing the degree of knockdown. Transfection performance was monitored using a validated MAPK1 siRNA (Hs/Mm MAPK1 control siRNA; Qiagen).

\section{RNA isolation}

RNA isolation and purification was performed using the 96-well RNeasy protocol (Qiagen), including the DNA removal step, according to the manufacturer's guidelines. RNA was eluted in $100 \mu \mathrm{L}$ of RNase-free water (Qiagen).

\section{qRT-PCR}

RNA samples were routinely diluted $1: 4$, and $5 \mu \mathrm{L}$ of the dilution was subjected to QuantiTect SYBR Green one-step qRT-PCR (Qiagen) according to the protocol 
given in the handbook. Final PCR volume was $20 \mu \mathrm{L}$ in 384-well plate format ( $25 \mu \mathrm{L}$ in 96 -well plates) containing $10 \mu \mathrm{L}(12.5 \mu \mathrm{L}$ in 96-well plates) $2 \times$ QuantiTect SYBR Green PCR Master Mix, $0.2 \mu \mathrm{L}(0.25 \mu \mathrm{L}$ in $96-$ well plates) QuantiTect Reverse Transcriptase Mix, and $0.6 \mu \mathrm{M}$ of each primer from the appropriate QuantiTect Primer Assay (Qiagen). Cycling conditions were $50^{\circ} \mathrm{C}$ for 30 minutes, $95^{\circ} \mathrm{C}$ for 15 minutes, followed by $45 \mathrm{cy}$ cles of $94^{\circ} \mathrm{C}$ for 15 seconds, $56^{\circ} \mathrm{C}$ for 30 seconds, $72^{\circ} \mathrm{C}$ for 30 seconds.

For two-step qRT-PCR analysis, $12 \mu \mathrm{L}$ RNA eluate and $2 \mu \mathrm{L}$ of $7 \times$ gDNA Wipeout Buffer were incubated for 2 minutes at $42^{\circ} \mathrm{C}$ and then chilled on ice. cDNA synthesis was performed by adding $1 \mu \mathrm{L}$ Quantiscript $\mathrm{Re}$ verse Transcriptase, $1 \mu \mathrm{L}$ RT Primer Mix, and $4 \mu \mathrm{L}$ of $5 \times$ Quantiscript RT Buffer and incubation at $42^{\circ} \mathrm{C}$ for 15 minutes (reaction components were from the QuantiTect Reverse Transcription Kit, Qiagen). After heat inactivation for 3 minutes at $95^{\circ} \mathrm{C}, \mathrm{PCR}$ analysis was performed by adding $5 \mu \mathrm{L}$ of a 1:5 cDNA dilution to $10 \mu \mathrm{L} 2 \times$ QuantiTect SYBR Green PCR Master Mix and 0.6 $\mu \mathrm{M}$ of each primer from the appropriate QuantiTect Primer Assay in a final volume of $20 \mu \mathrm{L}$. Cycling conditions were as described above, omitting the first reverse transcription step. PCR runs were performed on an ABI7900 (384-well) and a BioRad i-Cycler (96-well).

\section{Target expression analysis and testing of primer function}

The search for a suitable cell line and testing of primer function for each target was done as follows. Untransfected cells were seeded in a 96-well plate $\left(2 \times 10^{4}\right.$ cells per well, for HEK293, the cell number was $4 \times 10^{4}$ ) and grown under normal culture conditions. After 3 days, RNA was isolated as described above. Expression of each target and primer function were measured by either one-step or two-step qRT-PCR analysis in triplicate of 1:4 and 1:40 dilutions of the eluted RNA. A no template control (NTC) was analyzed in parallel.

\section{Knockdown quantification for siRNA validation}

Generally, at least two independent transfections were carried out per siRNA with two replicates each. This procedure was repeated once, resulting in eight knockdown values for each siRNA. Transfection performance was verified by analyzing the degree of silencing obtained with the positive control siRNA (targeting MAPK1) which was transfected in parallel on each plate. Effective transfection was defined as resulting in MAPK1 knockdown of $\geq 70 \%$. Plates displaying lower positive control knockdown efficiencies were not analyzed. A single PCR was performed for analysis of target and reference gene expression for each siRNA transfection. Samples of untransfected cells were analyzed in duplicate for both genes. Knockdown values were calculated by the $\Delta \Delta C_{T}$ method and GAPDH was used as an internal standard for normalization. Knockdown values were calculated with reference to untransfected cells. Validated siRNAs were defined as those with knockdown of at least $70 \%(\leq 30 \%$ mRNA remaining compared to untransfected cells). Generally the knockdown for a validated siRNA was the average of at least four knockdown values. $\Delta C_{T}$ s (no template control minus untransfected sample) were at least 6 for each target and each PCR run to exclude the possibility of contamination and to discriminate between primer dimers and small amplicons with low melting temperatures. Stringent specificity controls included melting curve analysis for each target mRNA amplification. This assured reliable amplification for each individual PCR reaction. PCR runs which did not meet these criteria were excluded from further analysis.

\section{RESULTS}

\section{Validation workflow}

A robust screening procedure has been established in order to address our two requirements of throughput and reliability of results. Huesken et al. (2005) have developed the BIOPREDsi algorithm using an artificial neural network which is based on an experimental data set of approximately 3000 siRNAs. This algorithm was used to design the siRNAs for the validation project.

A set of five commonly used human cell lines representing different tissues was chosen to ensure a broad spectrum of gene expression. The cell lines chosen were HeLa (cervix), HepG2 (liver), HEK293 (kidney), MCF-7 (breast), and A549 (lung). To avoid potential siRNA amount-dependant off-target effects which could hinder subsequent phenotypic assays (Persengiev et al., 2004; Semizarov et al., 2003; Sledz et al., 2003) we kept the siRNA concentration as low as possible for each cell line while still providing robust and reproducible results. Final siRNA concentrations of $5 \mathrm{nM}$ siRNA for HeLa and A549, or 25 nM siRNA for HEK293, HepG2, and MCF7 , were sufficient to obtain optimal results. Highthroughput, quantitative, real-time RT-PCR (qRT-PCR) was used for downstream analysis of the level of silencing. The whole experimental procedure was performed in a standardized manner using fixed procedures for transfection and qRT-PCR. Validation of each siRNA began with the selection of a suitable cell line with sufficient target gene expression, as determined by qRT-PCR analysis of untransfected cells. Next, siRNA was transfected and the mRNA knockdown level was determined 48 hours after transfection. Only those siRNAs that resulted in at least $70 \%$ knockdown (defined as mean value minus standard deviation) were defined as validated. 


\section{Knockdown efficiencies and reproducibility of validated siRNA}

To date, more than 3500 siRNAs targeting over 2000 genes have been validated. Gene targets cover a broad range of gene families including kinase and phosphatase genes as well as cancer-related genes involved in metastasis, DNA repair, tumor suppression, apoptosis, and cell cycle. The knockdown level achieved by the majority of the validated siRNAs ranged from $75 \%$ up to $89 \%$. The mean knockdown level achieved with validated siRNAs was $84 \%$ (Fig. 1). This demonstrates that silencing observed with many validated siRNAs was substantially better than the $70 \%$ threshold.

Generally, it is important for the success of an RNAi experiment that the siRNA is effective, and that its performance is reproducible. To investigate the reproducibility of siRNA performance in more detail, silencing data were randomly chosen and separated into two categories: 499 validated siRNAs with knockdown levels of at least $70 \%$ and 226 moderately functional siRNAs with knockdown levels of 50-69\%. The mean knockdown and the mean standard deviation were determined for the siRNAs in both groups. The mean knockdown for siRNAs in the first group was $84 \%$ with quite a low mean standard deviation of 6 , clearly reflecting their high silencing potency with only minimal variation. The mean knockdown level reached by the second group of siRNAs was $60 \%$, showing that a representative group of moderately functional siRNAs had been selected. In comparison with the validated siRNAs, variability was higher for these moderately functional siRNAs (a mean standard deviation of 14). siRNAs with a knockdown efficiency of $70 \%$ and greater thus appear to provide more robust and reproducible results when compared with moderately functional siRNAs.

\section{Target gene-inherent factors influence the gene silencing level}

One set of siRNAs which targets 625 kinases has been intensively investigated. The validation process started with testing two siRNAs per target. For those gene targets where only one or none of these two siRNAs were validated, we increased the number of siRNAs tested in steps, with the objective of identifying at least one and ideally two siRNAs per target. For the majority of targets in this set (79\%), two validated siRNAs were eventually identified. Between 3 and 5 validated siRNAs are available for $9 \%$ of the genes. For $6 \%$ of the target genes, only one siRNA tested showed at least $70 \%$ knockdown. The number of siRNAs that were tested in our attempt to reach our objective was different for each group of targets. For the vast majority (70\%), two out of the two initially tested siRNAs were validated (Fig. 2). Either 5-6 or 8-10 siRNAs were tested for $11 \%$ and $7 \%$ of the targets, respectively, to reach at least two validated siRNAs per target. As mentioned above, we identified more than two and in some cases up to five validated siRNAs for some targets by increasing the total number of siRNAs tested. In contrast, for $6 \%$ of the targets 9-10 siRNAs were tested to achieve one validated siRNA.

The mean knockdown obtained with this set of validated kinase siRNAs was $82 \%$, reflecting the average knockdown observed with all siRNAs validated so far. Figure 3 shows the distribution of knockdown of all siRNAs tested for the kinase targets for which at least one siRNA was finally validated. The majority of siRNAs $(67 \%)$ showed reproducible silencing of at least $70 \%$ and another $12 \%$ of siRNAs were close to our cutoff specification (showing knockdown between 50\% and 69\%) but did not pass our criteria, primarily due to variability of the knockdown achieved.

For $6 \%$ of the kinase target genes studied, none of the siRNAs tested resulted in over $70 \%$ silencing, even after testing up to 10 different siRNA sequences. Results obtained for two target genes of this group (diacylglycerol kinase epsilon (DGKE) and Rho GTPase activating protein 26 (ARHGAP26)) are shown in Figure 4. The majority of duplexes tested provided moderate to low knockdown levels. Only one siRNA for each target resulted in a knockdown level close to our cutoff specification. However, both of these siRNAs did not pass the validation criteria due to the standard deviation. For a proportion of unsuccessful kinase siRNAs, RISC competence was proven by cotransfection of each siRNA with a luciferase reporter plasmid. The corresponding siRNA-target sequences had been cloned into the 3' UTR of the luciferase gene. Knockdown was analyzed by luciferase assay two days after transfection. Preliminary data showed that four siRNAs which had resulted in only $20-60 \%$ knockdown of endogenously expressed gene targets gave improved knockdown levels of $80-90 \%$ when the target was recombinantly expressed (E. Lader and S. Yerramilli, personal communication). This result concurs with reports from Holen et al. (2002) and Schubert et al. (2005), who observed improved gene silencing of cotransfected recombinant genes compared with endogenously expressed genes. Taken together, these data indicate that targets differ in their susceptibility to siRNA-mediated knockdown.

To examine siRNA-related factors which could influence knockdown in more detail, siRNAs which showed either high knockdown (one siRNA each for targets MAPK1 and Ryk) or moderate/low knockdown (nine siRNAs each for targets DGKE and ARHGAP26) were analyzed. Apart from two siRNAs with GC contents of $21 \%$ and $63 \%$, all the siRNAs spanned a range of GC content of between $32 \%$ and $53 \%$. GC content did not correlate with the knockdown levels achieved using these siRNAs. 


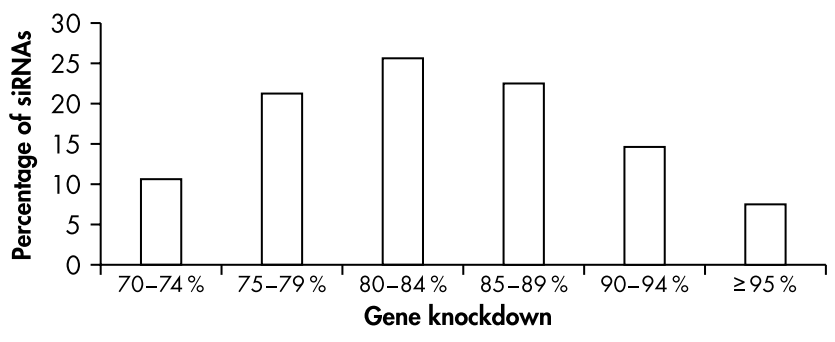

FIG. 1. Distribution of knockdown level achieved using validated siRNAs. The distribution of knockdown achieved using validated siRNAs shows that the knockdown level of a significant proportion of the validated siRNAs is higher than the $70 \%$ threshold level. The majority of validated siRNAs achieve knockdown levels in the range of $75-89 \%$. The mean knockdown value is $84 \%$.

To calculate predicted accessibility of the siRNA target sites, we used Sfold (Ding and Lawrence, 2001, 2003; Ding et al., 2004; http://sfold.wadsworth.org). We estimated the scores for the siRNAs using the siRNA binding site plus 40 adjacent nucleotides both upstream and downstream. The score provided by the software is the sum of various factors including target accessibility score, duplex feature score, and duplex thermodynamics score and can reach a maximum of 20 points. For the validated siRNAs scores of 9 (MAPK1) or 16 (Ryk) were achieved. For the 18 unsuccessful siRNAs (DGKE or ARHGAP26), scores of between 4 and 15 were achieved. For the majority of unsuccessful siRNAs $(60 \%)$, the score was within the range of 10-15. No significant correlation between knockdown achieved and Sfold scores was observed for the group of siRNAs examined.

\section{Influence of gene expression level}

Essential, highly expressed, and overexpressed genes can be efficiently silenced using siRNAs. However, it has been reported that low-abundant genes are less susceptible to siRNA-mediated knockdown (Hu et al., 2004). A sample of 1069 validated siRNAs was randomly chosen to investigate whether the expression level had an impact on the level of silencing achieved. The knockdown observed using these siRNAs was correlated with the gene expression level of the corresponding target genes. Analysis of gene expression for the target genes was performed by qRT-PCR of untransfected cells and the housekeeping gene glyceraldehyde-3-phosphate dehydrogenase (GAPDH) served as an internal reference. The $\Delta C_{T}$ values between the $C_{T}$ values of the target gene and the housekeeping gene GAPDH in untransfected cells were taken as a marker of gene expression. The target genes showed a broad range of gene expression levels (Fig. 5A). To allow detailed analysis, the siRNAs were divided into six groups representing knockdown levels of 70-74\%, 75-79\%, 80-84\%, 85-89\%, $90-94 \%$, or $\geq 95 \%$. The knockdown levels for all the validated siRNAs, even after division into the six groups, is normally distributed in each gene expression category showing efficient knockdown for moderately expressed as well as highly expressed genes (Fig. 5B). The same result was obtained for a smaller set of targets when siRNAs providing knockdown below $70 \%$ were included in the analysis (data not shown). This result shows that target genes with either high or moderate to low gene expression levels were silenced equally well. Therefore, target gene expression level does not serve as an indicator of successful silencing potential.
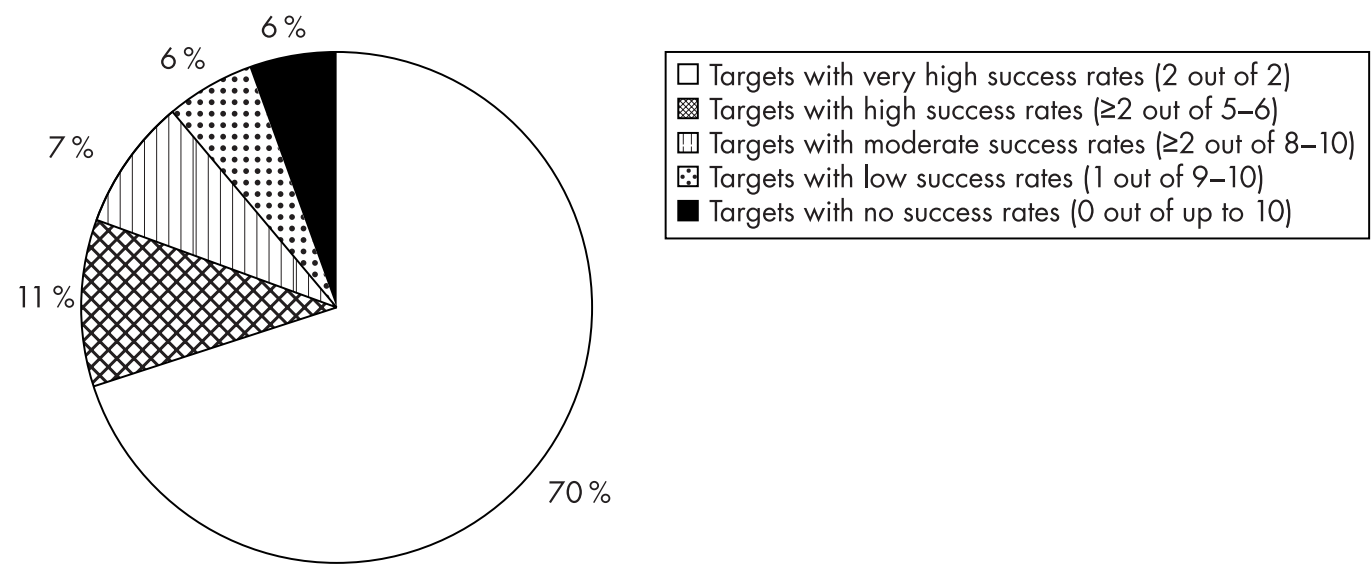

FIG. 2. Success rates of siRNAs targeting kinase genes. Kinase gene targets were divided into five groups according to the success rates observed with the siRNAs tested for these genes. For 70\% of targets, two out of two siRNAs initially tested were validated. Another $11 \%$ of targets showed high success rates when the number of siRNAs tested was increased (at least two siRNAs were validated after testing five to six siRNAs). Moderate to low success rates (either $\geq 2$ validated siRNAs out of $8-10$ siRNAs tested or one validated siRNA out of 9-10 siRNAs tested) were observed for siRNAs targeting 7\% and $6 \%$ of the genes, respectively. For the remaining $6 \%$ of targets, up to 10 siRNAs were tested without identifying any validated siRNAs. 


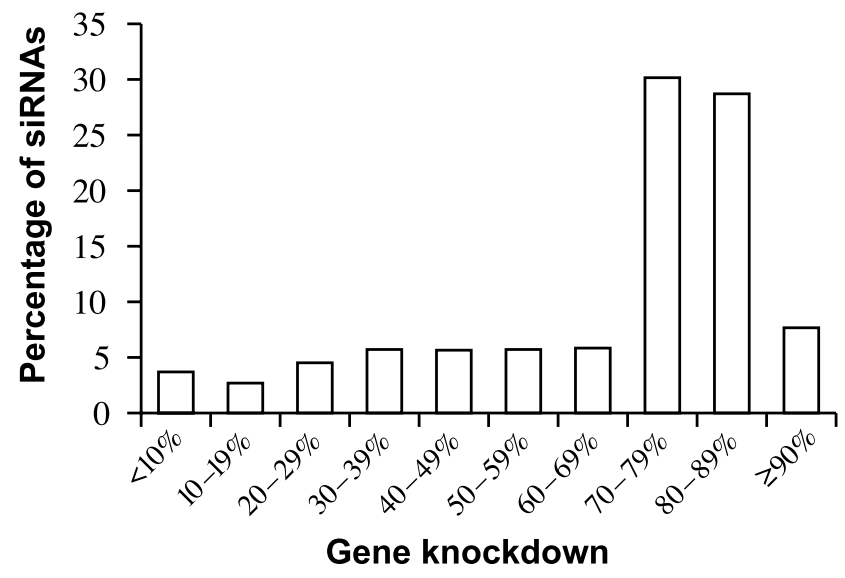

FIG. 3. Distribution of knockdown level achieved for kinase gene targets. Knockdown level of siRNAs for kinase targets for which at least one validated siRNA was found are shown. For the majority of siRNAs tested for these targets at least $70 \%$ of knockdown was achieved and a substantial fraction of siRNAs gave results higher than this threshold.

\section{Localization of siRNA target sites on the transcript}

Accessibility of the target site is critical for successful silencing. Activated RISC could compete with mRNA binding proteins for access to the corresponding target transcript sequences. The observation that silencing improves in the absence of translation (Gu and Rossi, 2005) supports this suggestion. We compared the target sequences of the siRNAs with their knockdown efficiencies to investigate whether siRNAs showing high knockdown are preferentially located in the $5^{\prime}$ untranslated region (UTR), within the coding sequence, or in the 3' UTR. Gene targets for which we have both validated siRNAs and less potent siRNAs showing moderate or low knockdown were chosen for this analysis. Both types of siRNA target sequence were homogenously distributed within the $3^{\prime}$ UTR, coding sequence, and 5' UTR (Fig. 6). This observation indicates that overall siRNA performance is similar for these three regions on the mRNA transcript.

\section{Impact of the siRNA concentration on the knockdown level}

To determine whether specific target-siRNA combinations could achieve improved knockdown if higher siRNA amounts were used, a range of siRNA concentrations (5-100 nM) was tested. A panel of targets was chosen for which at least one siRNA was validated, whereas another siRNA showed moderate or only poor silencing capacity.

A comparison of knockdown levels achieved using either $5 \mathrm{nM}$, our standard siRNA concentration for HeLa cells, or higher concentrations $(25 \mathrm{nM}, 50 \mathrm{nM}$, and 100 $\mathrm{nM}$ ) is shown for syntrophin associated serine/threonine kinase (SAST) in Figure 7. The 72\% silencing seen using $5 \mathrm{nM}$ of the validated siRNA (duplex 1) was only marginally improved when higher siRNA concentrations were tested. The moderate silencing efficiency obtained with the second siRNA (duplex 2) was also not significantly influenced by increasing the siRNA concentration. Similar results were observed with other siRNAs and target genes tested (data not shown). Only a few siRNA-target combinations showed slightly improved knockdown levels after use of $50 \mathrm{nM}$ or $100 \mathrm{nM}$ siRNA. In general, such high concentrations should be avoided to prevent concentration-dependent off-target effects (Persengiev et al., 2004; Semizarov et al., 2003; Sledz et al., 2003; ). Overall, we did not observe any significant improvement leading to reproducible silencing $>70 \%$ after using higher amounts of siRNA. The result indicates that use of high siRNA concentrations does not compensate for loss of target accessibility or low silencing capacity of an siRNA in the given experimental setting. This correlates with results reported by Kretschmer-Kazemi

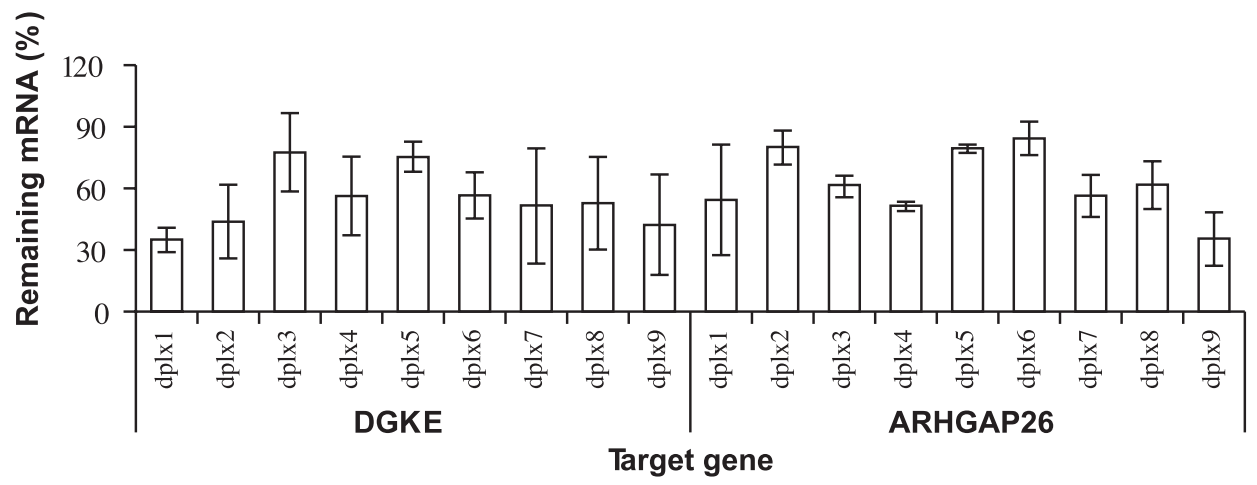

FIG. 4. Testing of siRNAs targeting difficult-to-silence genes. HeLa cells were transfected with $25 \mathrm{nM}$ of nine different siRNAs targeting either DGKE or ARHGAP26. Cells were harvested 48 hours after transfection. Each bar shows the average knockdown of eight transfections as determined by qRT-PCR. 
Far and Sczakiel (2003) obtained for two siRNAs addressing ICAM-1 suggesting that the functionality of siRNAs might be influenced by both concentration-dependent and concentration-independent parameters.

\section{Knockdown efficiency in various cell lines}

Apart from the siRNA sequence, characteristics inherent to the mRNA and cellular environment should be considered to achieve significant knockdown. Factors which may influence the target accessibility and thus the performance of an siRNA are the secondary or tertiary structure of a given mRNA and mRNA binding proteins which could either prevent activated RISC complexes binding their target sequences or could themselves influence the mRNA structure (Bohula et al., 2003; Brown et al., 2005; Heale et al., 2005; Kretschmer-Kazemi Far and Sczakiel, 2003; Schubert et al., 2005). Access to the mRNA might also be hampered by the cellular compartmentalization or the subcellular localization of the transcript. It is conceivable that accessibility of siRNA target sites varies between cell types. When working with continuously dividing cell lines, variations of target sequences due to spontaneous mutations or alternative splicing can occur, leading to discrepancies between the sequence in public databases and the actual transcript sequence in the cell line. Apart from sequence and target accessibility, cell types could differ in the organization of factors necessary for RNAi, especially of RISC. Many of these biological aspects cannot be satisfactorily addressed by design algorithms. To investigate a potential cell line-specific impact on the knockdown level, a set of target genes was chosen for which two siRNAs showed significant silencing capacity of at least $70 \%$ in HeLa cells. Additional cell lines which showed adequate gene expression for these target genes were selected based on the results of qRT-PCR analysis of untransfected cells. These cell lines were then transfected with the siRNAs using optimized transfection parameters. In line with the results observed in HeLa cells, significant knockdown levels of at least $70 \%$ were achieved in two other cell lines (Fig. 8).
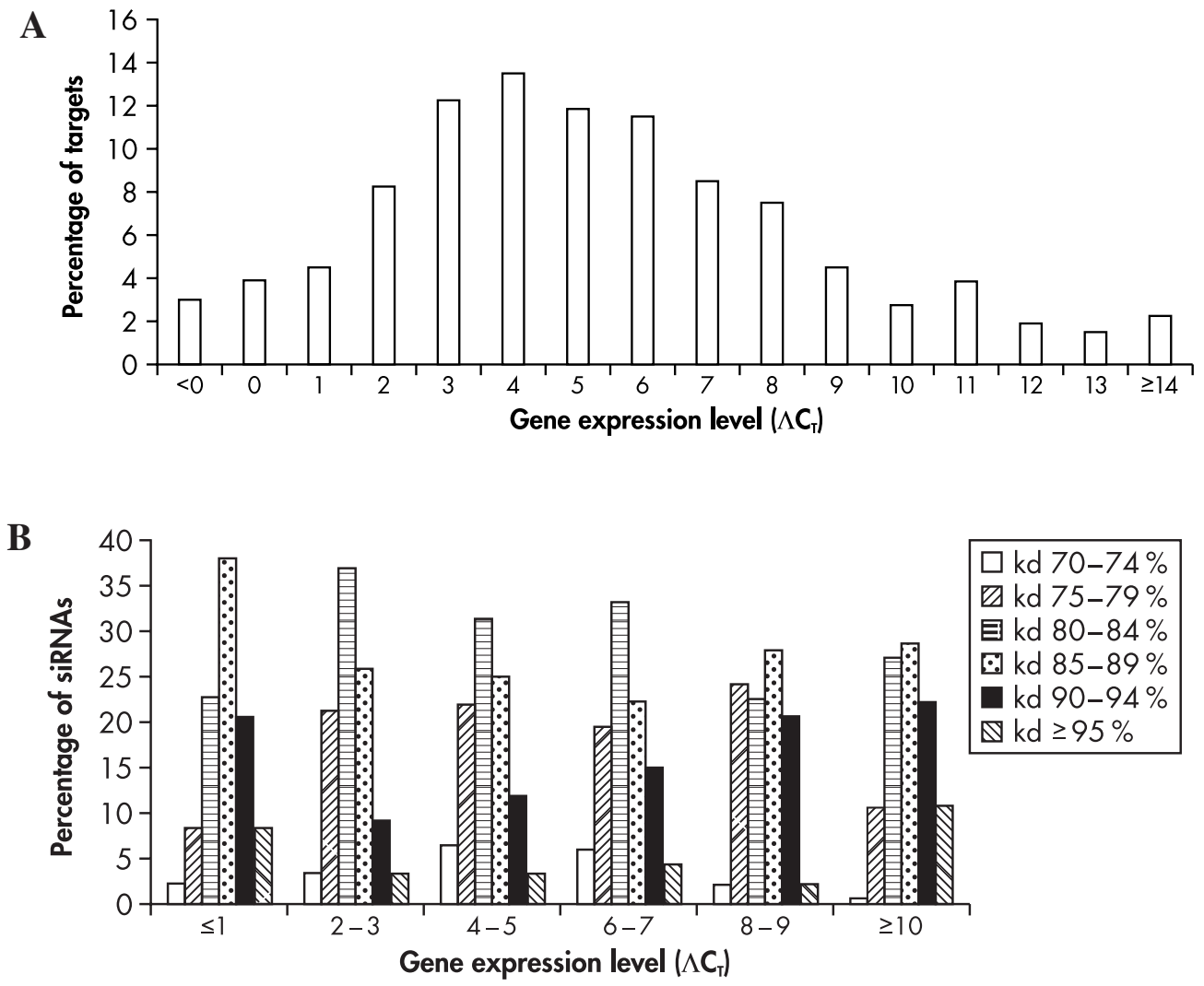

FIG. 5. Impact of gene expression level on knockdown. The knockdown level achieved with 1169 randomly chosen validated siRNAs was aligned with the gene expression level of the corresponding target genes. Gene expression was determined by qRTPCR of untransfected cells and normalized to the gene expression of the housekeeping gene GAPDH. The gene expression level, reflected by the corresponding $\Delta C_{T}$ value, spans a wide range including genes, which show low $\left(\Delta C_{T} \geq 14\right)$ or high $(\Delta C \mathrm{~T}<0)$ expression relative to GAPDH (A). A normal Gaussian distribution of the knockdown was obtained when analysing each of the gene expression categories separately $(\mathbf{B})$. 


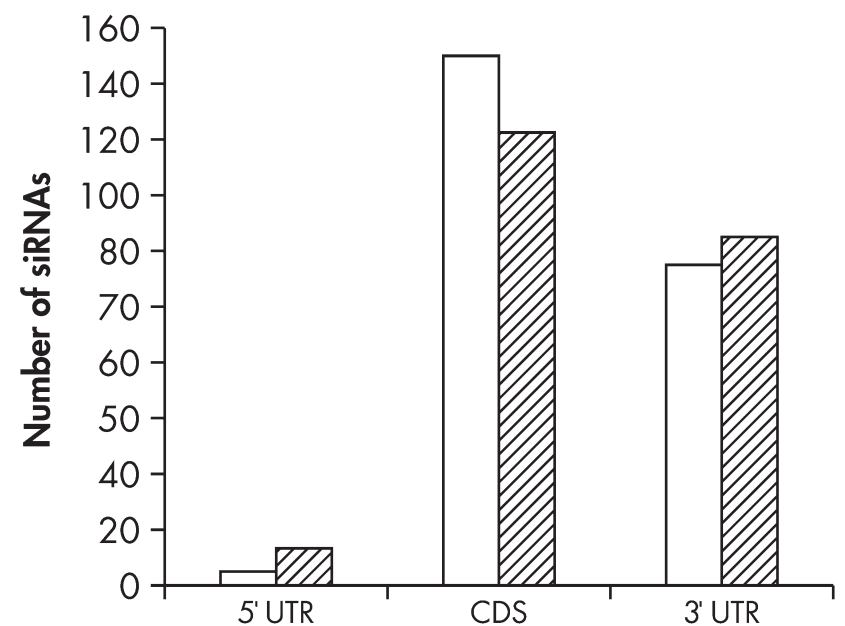

FIG. 6. Localization of the transcript sequence. The localization of target sequences for validated siRNAs and moderate or low silencing siRNAs within either the $5^{\prime}$ and $3^{\prime}$ untranslated region (5'UTR and $3^{\prime}$ UTR) or the coding sequence (CDS) was compared. The analysis is based on 228 randomly chosen validated siRNAs (white bars) and 217 siRNAs which showed less than $70 \%$ knockdown (hatched bars).

We observed that, for some targets, $\geq 70 \%$ knockdown at the mRNA level was difficult to achieve. Several kinase targets that fell into this category were investigated in more detail. Although up to 10 siRNAs were tested per gene, they all resulted in either moderate or low levels of silencing. As a high number of siRNAs were tested, this could not be a result of the siRNA sequences selected.

For a smaller subset of these kinase genes which were expressed in all five cell lines used in this study, potential cell-specific effects were studied more extensively. The majority of siRNAs showed comparable results in all the cell lines. siRNAs targeting diacylglycerol kinase epsilon
(DGKE) resulted in a moderate knockdown level in all five cell lines (Fig. 9). For two siRNAs targeting Rho GTPase activating protein 26 (ARHGAP26), low knockdown levels were obtained for both siRNAs in A549 and HEK293 cells. Silencing was only marginally higher in HepG2 (32-54\%) and HeLa (22-63\%). However, improved silencing of 60-70\% was observed after transfection into MCF-7 cells which, due to the standard deviation observed with both siRNAs, still did not fulfill the $70 \%$ criteria for validated siRNAs. Cell-specific differences were more pronounced for siRNAs targeting cyclin-dependent kinase 10 (CDK10). Both siRNAs showed almost no silencing in HEK293 cells. Improved silencing was observed in A549 cells with moderate silencing achieved for duplex $1(42-44 \%)$ and $64 \%-68 \%$ knockdown achieved for duplex 2. Best results for both siRNAs were achieved in HepG2 and MCF-7 cells. Knockdown for both duplexes was close to $70 \%$ in MCF7 and 84-88\% silencing was obtained using duplex 2 in HepG2, thus fulfilling our criteria for validated siRNAs. Together, these results and results from additional target-siRNA combinations investigated showed that the knockdown data obtained in HeLa cells were reproducible in the other cell lines used for validation. In only a few cases did changing the cell line result in improved silencing bringing the silencing observed close to our $70 \%$ criteria for validated siRNAs. Significant cell-specific changes in silencing were an exception. In conclusion, we did not observe a cell-specific impact on knockdown in the five cell lines investigated with either validated siRNAs or with siRNAs showing moderate to low silencing. This agrees with data reported by Bohula et al. (2003) for two siRNAs targeting type insulin-like growth factor receptor (IGF1R) and with data from cotransfections of siRNA with reporter constructs (Holen et al., 2002).

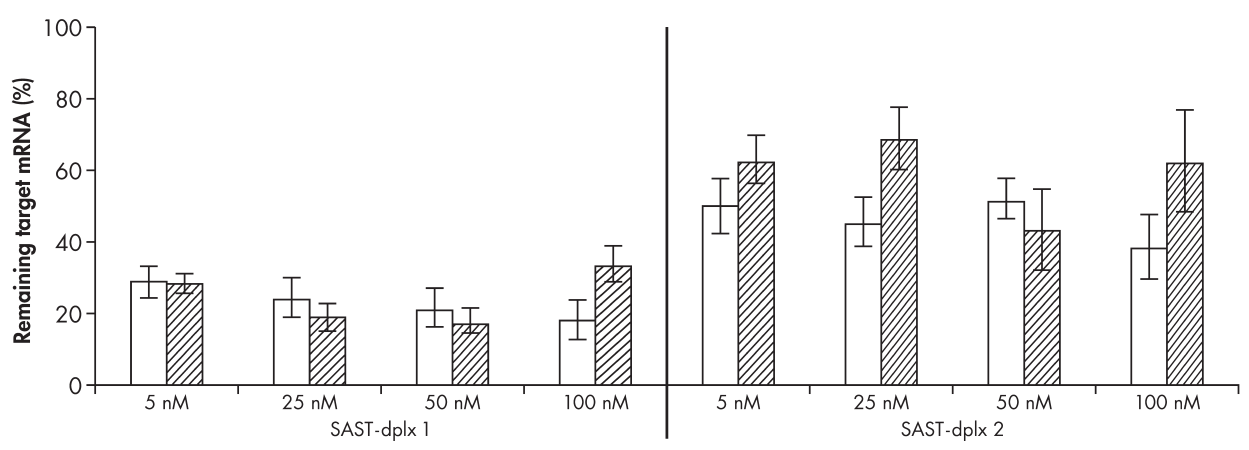

FIG. 7. Applying increased siRNA concentrations. In two independent experiments, HeLa cells were transfected with the indicated concentrations of siRNAs targeting SAST. For 50 and $100 \mathrm{nM}$ concentrations, $1.5 \mu \mathrm{L}$ HiPerFect Transfection Reagent was used. The other transfections were performed as described in Materials and Methods. Duplex 1 was validated during the validation project and duplex 2 did not meet the 70\% knockdown criteria. Cells were harvested 48 hours post-transfection. Each bar shows the average knockdown of four transfections determined by qRT-PCR. The results of two independent experiments are shown. 


\section{Kinetics of knockdown}

Knockdown level was analyzed on day 2 after transfection, which was appropriate for the majority of siRNAs tested. However, as some target genes may be optimally silenced at a later or earlier time point, this possibility was investigated further. Kinase target genes that showed only moderate or low knockdown levels after transfection of several different siRNAs into HeLa cells were chosen for this investigation. We tested whether the level of silencing was influenced by shortened or prolonged incubation times of either 24 or 72 hours after transfection.

Representative results for two siRNA duplexes targeting kinase targets DGKE and ARHGAP26 are shown in Figure 10. A validated siRNA targeting the RYK receptor-like tyrosine kinase (RYK) served as an internal control. Comparable results of 73-79\% were achieved for the validated RYK siRNA, independent of the time point of analysis. The silencing levels achieved using siRNAs targeting both other genes were low or moderate but below $70 \%$ knockdown 48 hours after transfection. For one target (DGKE), optimal results at the mRNA level (53\% and $47 \%$ knockdown) were achieved when cells were analyzed 72 hours after transfection. This level was still well below the $70 \%$ required for a validated siRNA. The knockdown levels reached for both siRNAs targeting the gene ARHGAP26 were similar at 48 and 72 hours after transfection and these levels were clearly higher compared with data obtained 24 hours after transfection.
However, the knockdown achieved was still below $70 \%$. No significant improvement in silencing was observed for these examples after shortened or prolonged incubation times. These results are representative of those observed with other targets tested (data not shown). In general, incubation times of 24 hours after transfection were short for certain genes, whereas an increase in incubation time from 48 hours up to 72 hours had only slight or negligible effects on the silencing level. In cell types with short doubling times, longer incubation could lead to reduction of the knockdown observed at the mRNA level due to dilution of the siRNA during cell proliferation (Bartlett and Davis, 2006; Layzer et al., 2004; Song et al., 2003; Tuschl, 2002). Our results show that analysis on day 2 after transfection is suitable for knockdown analysis, especially when working with a broad variety of target genes.

Further modifications of the procedure were investigated, especially with siRNAs showing moderate or low silencing levels under the experimental conditions used. Cells could counteract loss of mRNA induced by gene silencing with enhanced mRNA transcription in an attempt to maintain a normal level of mRNA transcripts. We investigated whether a second boost of siRNA would compensate for already depleted siRNA. HeLa cells were transfected twice with an incubation time of 24 hours between both transfections. Overall, no significant improvement of knockdown was observed after serial transfection of cells when compared with single transfections (data not shown). Silencing was also comparable for ei-

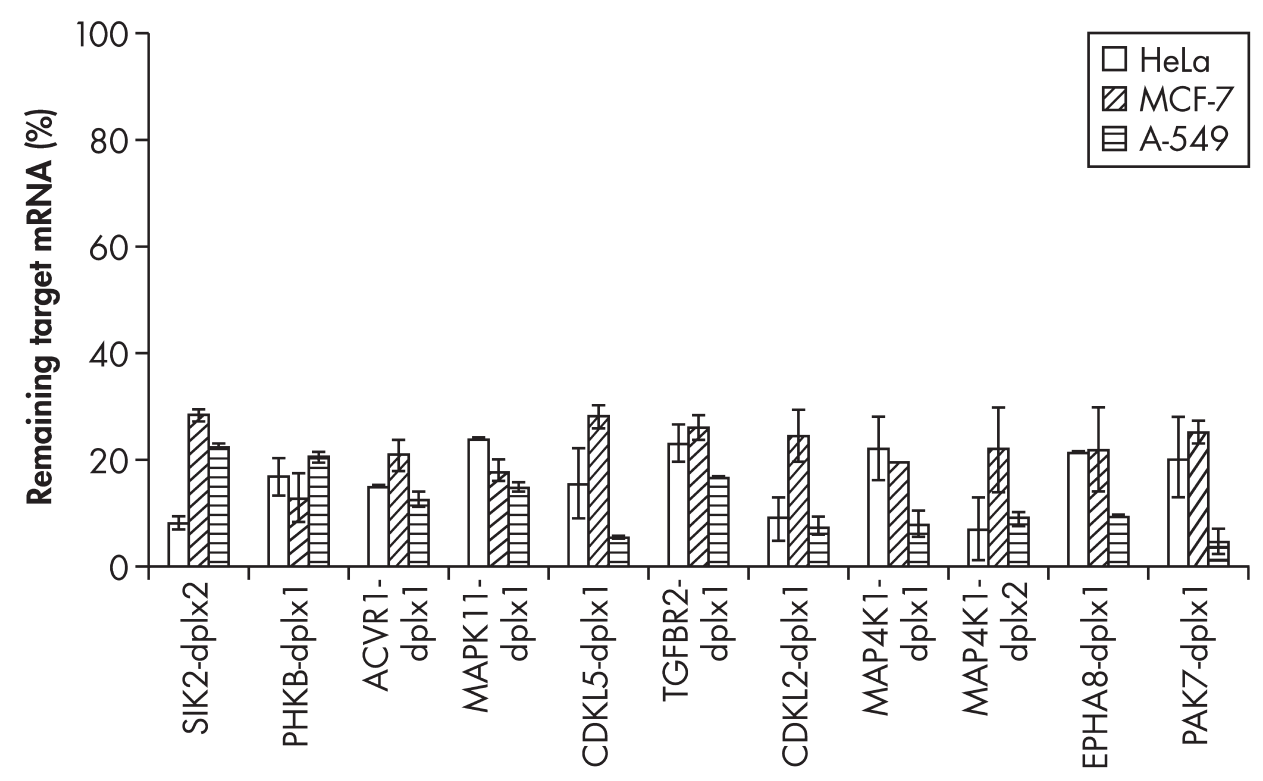

FIG. 8. Knockdown efficiency in multiple cell lines. Gene knockdown using validated siRNAs was determined in three different cell lines. HeLa, MCF-7, and A549 cells were transfected with the indicated siRNAs using transfection conditions as described in Materials and Methods. Cells were harvested 48 hours post-transfection. Each bar shows the average knockdown from four transfections as determined by qRT-PCR. 


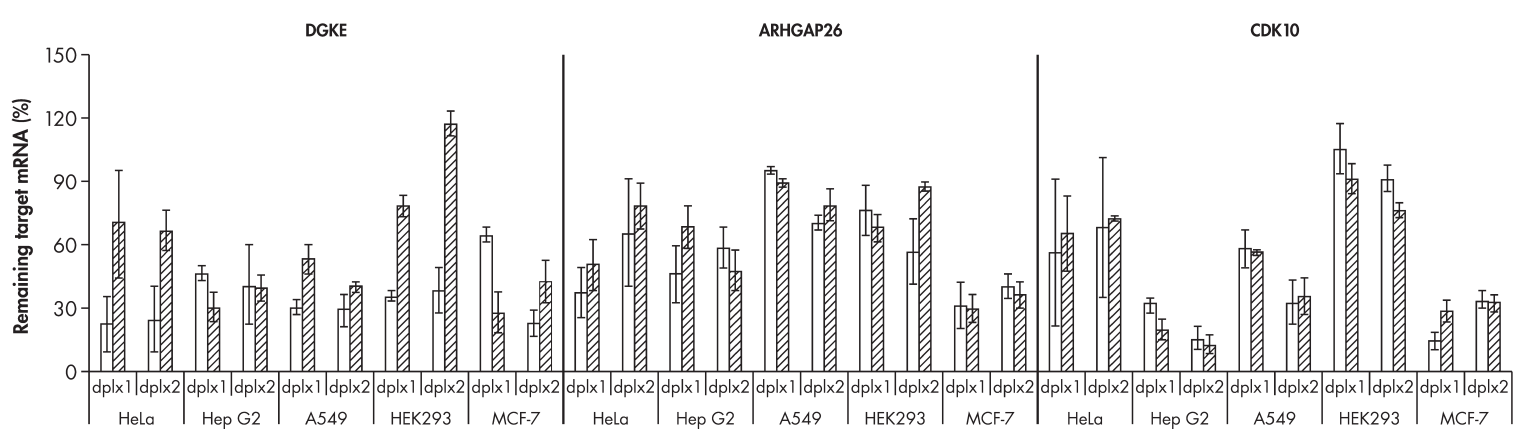

FIG. 9. Determination of siRNA functionality in different cell lines. HeLa, HepG2, A549, HEK293, and MCF-7 cells were transfected with the indicated siRNAs using siRNA concentrations and transfection conditions as described in Materials and Methods. Cells were harvested 48 hours post-transfection. Each bar shows the average knockdown from four transfections as determined by qRT-PCR. The results of two independent experiments are shown.

ther individual or simultaneous transfection of two siRNAs showing only moderate to low knockdown (data not shown), excluding a potential synergistic or additive effect due to reciprocal facilitating access to the mRNA.

These results, together with the kinetic and comparison data generated in several cell lines, suggest that other target or mRNA-inherent aspects must be limiting in the case of difficult-to-knockdown targets.

\section{DISCUSSION}

Validating several thousand siRNAs for various target genes using a single design algorithm and a standardized validation procedure provided us with a huge, homogeneous data set which we took as a basis for investigating factors critical for effective gene silencing. All data are based on knockdown of endogenously expressed genes and thus reflect the natural characteristics such as structure and localization of a given target mRNA in the cellular environment.

The silencing level achieved is dependant on various factors, each reflecting the different steps of a gene si- lencing experiment. In recent years, rational design tools have been developed which design specific and high-performing siRNA sequences. Working with such a design tool, the BIOPREDsi algorithm, allowed us to validate several thousand siRNAs, many of which provided silencing levels far $>70 \%$. Nevertheless, some genes proved less amenable to siRNA-mediated silencing and the $70 \%$ knockdown threshold was difficult to achieve. In some cases, up to 10 different siRNAs tested failed to reproducibly provide at least $70 \%$ knockdown. In contrast, for other target genes, two out of two initially tested siRNAs provided knockdown of $70 \%$ and higher. Those siRNAs, which were validated in HeLa cells, provided silencing at a similar level in other cell lines where the transfection efficiencies and gene expression levels were comparable. This result showed that the validation data obtained were highly transferable between cell lines, which is especially relevant when using these siRNAs for further loss-of-function studies.

The performance of siRNAs showing moderate silencing of $<70 \%$ was almost unaffected by increasing the siRNA concentration used for transfection, by changing the cell type, or by increasing or decreasing the time

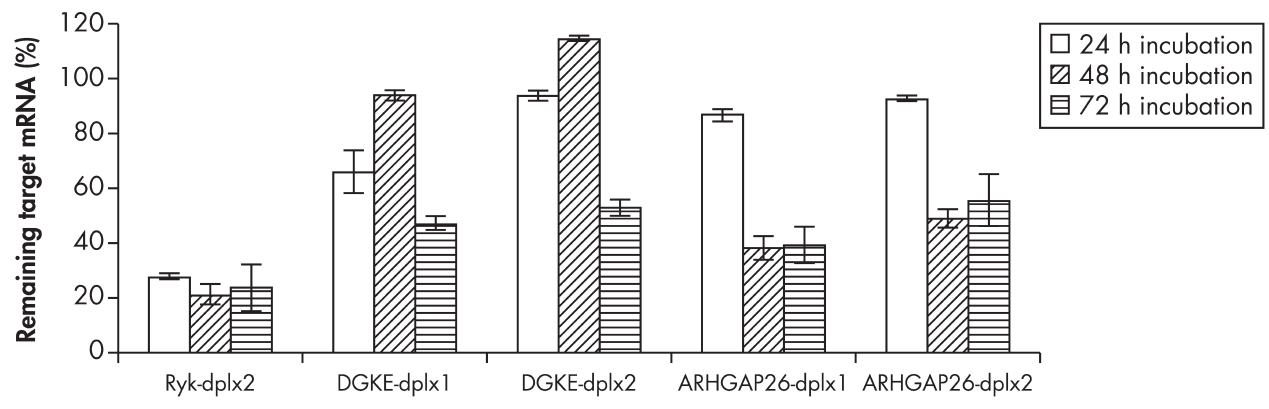

FIG. 10. Kinetic analysis of siRNA-mediated knockdown. HeLa cells were transfected with the indicated siRNAs. Cells were harvested 24, 48, and 72 hours post-transfection. Each bar shows the average knockdown from four transfections as determined by qRT-PCR. 
point of analysis. Limitations caused by either the siRNA sequences themselves (e.g., suboptimal incorporation into RISC) or by target-inherent aspects (e.g., accessibility of the target sequence, factors involved in binding and subsequent cleavage of the activated RISC) which have not been addressed with these parameters could be an explanation for this observation. Overall, the results obtained with both validated and moderately functional siRNAs showed that the conditions used for validation were suitable to ensure selection of effective siRNAs which provide reliable and sufficient knockdown.

The finding that targets differ with respect to the success rates of siRNAs tested and especially observations made of targets showing moderate or low silencing after transfection of several siRNAs indicate that target-inherent factors have a substantial impact on the success of a gene silencing experiment. This finding was initially made for a set of siRNAs targeting kinase genes which have been intensively investigated. However, target differences were not confined to this set but also observed with the various gene families included in the validation study. We excluded a correlation between gene expression level and the knockdown achieved, finding instead that efficient silencing levels were achieved using siRNAs for genes that were normally distributed over a broad range of gene expression levels. The activated RISC has a multiturnover characteristic which may therefore be similarly advantageous for silencing both high and low transcript levels.

Effective siRNA delivery is a prerequisite for efficient silencing. The optimal transfection conditions for a given cell type are dependent on the siRNA concentration in combination with the transfection reagent used. We found that silencing achieved with our standard condition of $5 \mathrm{nM}$ in HeLa cells was not improved by increasing the siRNA concentration. We made this observation for both validated siRNAs as well as siRNAs showing moderate or low silencing. Once transfection conditions had been optimized, the silencing level achieved with a given siRNA-target gene combination was not significantly improved by increased siRNA amount. In the case of siRNAs showing moderate or low silencing ability, limitations therefore arise from concentration-independent aspects of the gene-silencing process (KretschmerKazemi Far and Sczakiel, 2003).

The siRNA design algorithm can ensure effective incorporation of the selected siRNA into RISC and efficient and specific cleavage of the target sequence upon binding of the activated RISC. However, secondary and tertiary structures of the mRNA, binding proteins, and the subcellular localization of the transcript may hamper or even prevent access of activated RISC to the corresponding target sequences, leading to reduced silencing or completely blocking silencing. Alignment of either validated or moderately functional siRNAs with the cor- responding target sequence on the mRNA showed that both are homogenously distributed within the $5^{\prime}$ UTR, the coding sequence, and the $3^{\prime}$ UTR. Based on these results, we could exclude a direct correlation between the localization of the siRNA target sequence and the silencing level reached. This is in agreement with data published by others (Harborth et al., 2003; Hsieh et al., 2004; Overhoff et al., 2005), which has shown comparable silencing for siRNAs directed against the 3' UTR, 5' UTR, and the coding sequence. Selecting siRNAs that target specific regions on the transcript does not appear to offer any advantage.

In principle, detailed analysis of a given mRNA would be valuable information for the selection of accessible target sites which could be used for the design of potent siRNAs. Investigations using computational tools that predict the structure of an mRNA sequence or selection of suitable target sites experimentally using RNase $\mathrm{H}$ mapping data have confirmed that the mRNA structure has an impact on the overall performance (Bohula et al., 2003; Kawasaki et al., 2003; Overhoff et al., 2005; Vickers et al., 2003). However, these tools provide only limited cognitions, which are not sufficient to reliably predict efficient siRNAs. One major reason for this may be the difficulty of adequately reflecting the cellular environment. In line with this observation, we did not find a correlation between knockdown and Sfold scoring when comparing validated siRNAs with siRNAs for difficultto-silence target genes that showed moderate to low silencing.

By comparing knockdown efficiencies of multiple siRNAs for one target, it has been shown that altering the corresponding target sequences by even 1 nucleotide can have a huge effect on the silencing level achieved using a given siRNA (Ui-Tei et al., 2004). This demonstrates that other factors, apart from the accessibility of the target site, are essential for effective knockdown.

Design and performance of siRNAs is strictly dependent on the accuracy of the sequence information for the target gene. Since sequence information is constantly evolving, up-to-date information on the target genes available in public domain, including known transcript variants and single nucleotide polymorphisms (SNPs), have been taken into account when designing siRNAs and primers used for downstream qRT-PCR analysis. Cell-specific sequence discrepancies (e.g., changes in sequence caused by sporadically occurring mutations) may emerge and accumulate in cell cultures, especially in continuously dividing cancer cells (Huppi et al., 2005). In addition, potential competition between siRNA and endogenously expressed miRNA could lead to cell-specific differences when silencing with identical siRNAs. By comparing three cell lines, we showed that the knockdown level achieved with validated siRNAs was independent of the cell line used. The same result was ob- 
served with the majority of siRNAs which showed moderate knockdown. Together, these results demonstrate that the overall structure and subcellular localization of the mRNA of the target-siRNA combinations tested is similar in all the cell lines investigated. The corresponding target sites are either fully or partially accessible in the case of validated siRNAs and moderately functional siRNAs respectively, or are inaccessible to siRNA binding in the case of nonfunctional siRNAs. These data also imply that the five cell lines used share a similar set of factors necessary for RNAi, such as the RISC components.

In summary, comparable results were observed with a variety of siRNAs that showed either silencing of $70 \%$ and over or moderate knockdown levels below this threshold. Apart from reduced reproducibility observed in the second group, the knockdown levels achieved for both groups was independent of the cell type and the target gene expression level. Both types of siRNAs responded similarly to changes in siRNA concentration and the time point of analysis. These results demonstrate the transferability of results obtained for a particular siRNA sequence. In addition, they show target-inherent differences in susceptibility to siRNA-mediated gene silencing. Emerging knowledge on the factors necessary for efficient RNAi and the target gene-specific characteristics that facilitate RNAi, as well as further investigation into the interactions of the cellular components involved, will undoubtedly improve the ability to predict siRNA functionality and performance.

\section{ACKNOWLEDGMENTS}

We thank Eric Lader and Subrahmanyam Yerramilli for designing the siRNAs and performing RISC-competence experiments in their research group. We also thank Elizabeth Scanlan for reading the manuscript.

\section{REFERENCES}

ARONIN, N. (2006). Target selectivity in mRNA silencing. Gene Ther. 13, 509-516.

BARTLETT, D.W., and DAVIS, M.E. (2006). Insights into the kinetics of siRNA-mediated gene silencing from live-cell and live-animal bioluminescent imaging. Nucleic Acid Res. 34, 322-333.

BOHULA, E.A., SALISBURY, A.J., SOHAIL, M., PLAYFORD, M.P., RIEDEMANN, J., SOUTHERN, E.M., and MACAULAY, V.M. (2003). The efficacy of small interfering RNAs targeted to the Type 1 Insulin-like growth factor receptor (IGF1R) is influenced by secondary structure in the IGF1R transcript. J. Biol. Chem. 278, 15991-15997.

BROWN, K.M., CHU, C-Y., and RANA, T.M. (2005). Target accessibility dictates the potency of human RICS. Nat. Struct. Mol. Biol. 12, 469-470.
CAPLEN, N.J., PARRISH, S., IMANI F., FIRE, A., and MORGAN, R.A. (2001). Specific inhibition of gene expression by small double-stranded RNAs in invertebrate and vertebrate systems, Proc. Natl. Acad. Sci. USA 98, 9742-9747.

COLLINS, R.E., and CHENG, X. (2005). Structural domains in RNAi. FEBS Lett. 579, 5841-5849.

DING, Y., and LAWRENCE, C.E. (2003). A statistical sampling algorithm for RNA secondary structure prediction. Nucleic Acids Res. 31, 7280-7301.

DING, Y., and LAWRENCE, C.E. (2001). Statistical prediction of single-stranded regions in RNA secondary structure and application to predicting effective antisense target sites an beyond, Nucleic Acids Res. 29, 1034-1046.

DING, Y., CHAN, C.Y., and LAWRENCE, C.E. (2004). Sfold web server for statistical folding and rational design of nucleic acids. Nucleic Acids Res. 32(Web Server Issue), W135-W141.

DYKXHOORN, D.M., NOVINA, C.D., and SHARP, P.A. (2003). Killing the messenger: short RNAs that silence gene expression. Nat. Rev. Mol. Cell Biol. 4, 457-467.

ELBASHIR, S.M., LENDECKEL, W., and TUSCHL, T. (2001a). RNA interference is mediated by 21- and 22-nucleotiode RNAs. Genes Dev. 15, 188-200.

ELBASHIR, S.M., HARBORTH, J., LENDECKEL, W., YALCIN, A., WEBER, K., and TUSCHL, T. (2001b). Duplexes of 21-nucleotide siRNAs mediate RNA interference in cultured mammalian cells. Nature 411, 494-498.

FILIPOWICZ, W. (2005). RNAi: the nuts and bolts of the RISC machine. Cell 122, 17-20.

FIRE, A., XU, S., MONTGOMERY, M.K., KOSTAS, S.A., DRIVER, S.E., and MELLO, C.C. (1998). Potent and specific genetic interference by double-stranded RNA in Caenorhabditis elegans. Nature 391, 806-811.

GU, S., and ROSSI, J.J. (2005). Uncoupling of RNAi from active translation in mammalian cells. RNA 11, 38-44.

HARBORTH, J., ELBASHIR, S.M., VANDENBURGH, K., MANNINGA, H., SCARINGE, S.A., WEBER, K., and TUSCHL, T. (2003). Sequence, chemical, and structural variation of small interfering siRNAs and short hairpin RNAs and the effect on mammalian gene silencing. Antisense Nucleic Acid Drug Dev. 13, 83-105.

HEALE, B.S.E., SOIFER, H.S., BOWERS, C., and ROSSI, J.J. (2005). siRNA target site secondary structure predictions using local stable substructures. Nucleic Acids Res. 33, e30.

HAMILTON, A.J., and BAULCOMBE, D.C. (1999). A species of small antisense RNA in posttranscriptional gene silencing in plants. Science 286, 950-952.

HAMMOND, S.M. (2005). Dicing and slicing: the core machinery of the RNA interference pathway. FEBS Lett. 579, 5822-5829.

HAMMOND, S.M., BERNSTEIN, E., BEACH, D., and HANNON, G.J. (2000). An RNA-directed nuclease mediates posttranscriptional gene silencing in Drosophila cells. Nature 404, 293-296.

HANNON, G.J. (2002). RNA interference. Nature 418, 244-251.

HOLEN, T., AMARZGUIOUI, M., WIIGER, M.T., BABAIE, E., and PRYDZ, H. (2002). Positional effects of short interfering RNAs targeting the human coagulation trigger tissue factor. Nucleic Acids Res. 30, 1757-1766. 
HSIEH, A.C., BO, R., MANOLA, J., VAZQUEZ, F., BARE, O., KHVOROVA, A., SCARINGE, S., and SELLERS, W.R. (2004). A library of siRNA duplexes targeting the phosphoinositide 3-kinase pathway: determinants of gene silencing for use in cell-based screens. Nucleic Acid Res. 32, 893-901.

HU, X., HIPOLITO, S., LYNN, R., ABRAHAM, V., RAMOS, S., and WONG-STAAL, F. (2004). Relative gene-silencing efficiencies of small interfering RNAs targeting sense and antisense transcripts from the same genetic locus. Nucleic Acid Res. 32, 4609-4617.

HUESKEN, D., LANGE, J., MICKANIN, C., WEILER, J., ASSELBERGS, F., WARNER, J., MELOON, B., ENGEL, S., ROSENBERG, A., COHEN, D., LABOW, M., REINHARDT, M., NATT, F., and HALL, J. (2005). Design of a genome-wide siRNA library using an artificial neural network. Nat. Biotechnol. 23, 995-1001.

HUPPI, K., MARTIN, S.E., and CAPLEN, N.J. (2005). Defining and assaying RNAi in mammalian cells. Mol. Cell 17, $1-10$.

JAGLA, B., AULNER, N., KELLY, P.D., SONG, D., VOLCHUK, A., ZATORSKI, A., SHUM, D., MAYER, T., DE ANGELIS, D.A., OUERFELLI, O., RUTISHAUSER, U., and ROTHMAN, J.E. (2005). Sequence characteristics of functional siRNAs. RNA 11, 864-872.

KAWASAKI, H., SUYAMA, E., IYO, M., and TAIRA, K. (2003). siRNAs generated by recombinant DICER induce specific and significant but target site-independent gene silencing in human cells. Nucleic Acid Res. 31, 981-987.

KHVOROVA, A., REYNOLDS, A., and JAYASENA, S.D. (2003). Functional siRNAs and miRNAs exhibit strand bias. Cell 115, 209-216.

KRETSCHMER-KAZEMI FAR, R., and SCZAKIEL, G. (2003). The activity of siRNA in mammalian cells is related to structural target accessibility: a comparison with antisense oligonucleotides. Nucleic Acid Res. 31, 4417-4424.

LAYZER, J.M., MCCAFFREY, A.P., TANNER, A.K, HUANG, Z., KAY, M.A., and SULLENGER, B.A. (2004). In vivo activity of nuclease-resistant siRNAs. RNA 10, 766-771.

MIYAGISHI, M., and TAIRA, K. (2005). siRNA becomes smart and intelligent. Nat. Biotechnol. 23, 946-947.

OVERHOFF, M. , ALKEN, M., KRETSCHMER-KAZEMI FAR, R., LEMAITRE, M., LEBLEU, B., SCZAKIEL, G., and ROBBINS, I. (2005). Local RNA target structure influences siRNA efficacy: a systematic global analysis. J. Mol. Biol. 348, 871-881.

PERSENGIEV, S., ZHU, X., and GREEN, M.R. (2004). Nonspecific, concentration-dependent stimulation and repression of mammalian gene expression by small interfering RNAs (siRNAs). RNA 10, 12-18.

REYNOLDS, A., LAEKE, D., BOESE, Q., SCARINGE, S., MARSHALL, W.S., and KHVOROVA, A. (2004). Rational siRNA design for RNA interference. Nat. Biotechnol. 22, 326-330.
SCHUBERT, S. GRÜNWELLER, A., ERDMANN, V.A., and KURRECK, J. (2005). Local RNA target structure influences siRNA efficacy: systematic analysis of intentionally designed binding regions. J. Mol. Biol. 348, 883-893.

SCHWARZ, D.S., HUTVÁGNER, G., DU, T., XU, Z., ARONIN, N., and ZAMORE, P.D. (2003). Asymmetry in the assembly of the RNAi enzyme complex. Cell 115, 199-208.

SEMIZAROV, D., FROST, L., SARTHY, A., KROEGER, P., HALBERT, D.N., and FESIK, S.W. (2003). Specificity of short interfering RNA determined through gene expression signatures. Proc. Natl. Acad. Sci. USA 100, 6347-6352.

SLEDZ, C.A., HOLKO, M., DE VEER, M.J., SILVERMAN, R.S., and WILLIAMS, B.R.G. (2003). Activation of the interferon system by short-interfering RNAs. Nat. Cell Biol. 5, 834-839.

SONG, E., LEE, S.-K., DYKXHOORN, D.M., NOVINA, C., ZHANG, D., CRAWFORD, K., CERNY, J., SHARP, P.A., LIEBERMAN, J., MANJUNATH, N., and SHANKAR, P. (2003). Sustained small interfering RNA-mediated human immunodeficiency virus type 1 inhibition in primary macrophages. J. Virol. 77, 7174-7181.

TUSCHL, T. (2002). Expanding small RNA interference. Nat. Biotechnol. 20, 446-448.

TUSCHL, T., ZAMORE, P.D., LEHMANN, R., BARTEL, D.P., and SHARP, P.A. (1999). Targeted mRNA degradation by double-stranded RNA in vitro. Genes Dev. 13, 3191-3197.

UI-TEI, K., NAITO, Y., TAKAHASI, F., HARAGUCHI, T., OHKI-HAMAZAKI, H., JUNI, A., UEDA, R., and SAIGO, K. (2004). Guidelines for the selection of highly effective siRNA sequences for mammalian and chick RNA interference. Nucleic Acids Res. 32, 936-948.

VICKERS, T.A., KOO, S., BENNETT, C.F., CROOKE, S.T., DEAN, N.M., and BAKER, B.F. (2003). Efficient reduction of target RNAs by small interfering RNA and RNase H-dependent antisense agents. J. Biol. Chem. 278, 71087118.

ZAMORE, P.D., and HALEY, B. (2005). Ribo-gnome: the big world of small RNAs. Science 309, 1519-1524.

ZAMORE, P.D., TUSCHL, T., SHARP, P.A., and BARTEL, D.P. (2000). RNAi: double stranded RNA directs the ATPdependent cleavage of mRNA at 21 to 23 nucleotide intervals. Cell 101, 25-33.

Address reprint requests to: Ute Krüger, Ph.D. Senior Scientist Cell Biology QIAGEN GmbH QIAGEN Strasse 1 40724 Hilden, Germany

E-mail: ute.krueger@qiagen.com 
SupPlementary TABle 1

\begin{tabular}{|c|c|c|c|}
\hline Gene symbol & siRNA duplex & Entrez gene ID & siRNA target sequence \\
\hline ACVR1 & ACVR1-dplx1 & 90 & CGGATGGTGAGCAATGGTATA \\
\hline ARHGAP26 & ARHGAP26-dplx1 & 23092 & ACGGATACGGATGATTGAGAA \\
\hline ARHGAP26 & ARHGAP26-dplx2 & 23092 & АCTGCAАCССААССТАСТСТА \\
\hline ARHGAP26 & ARHGAP26-dplx3 & 23092 & CAGGACCAAGACAGAACTCAA \\
\hline ARHGAP26 & ARHGAP26-dplx4 & 23092 & СТССТТССТАТTAGСТАСАAА \\
\hline ARHGAP26 & ARHGAP26-dplx5 & 23092 & CTGGAATICCTIGTTAGGGAA \\
\hline ARHGAP26 & ARHGAP26-dplx6 & 23092 & ACCGTATGGCCTATAGTTTAA \\
\hline ARHGAP26 & ARHGAP26-dplx7 & 23092 & AGGGAGTATACTAGTAGGTTA \\
\hline ARHGAP26 & ARHGAP26-dplx8 & 23092 & CACCCTAGTAACCTCTTGAGA \\
\hline ARHGAP26 & ARHGAP26-dplx9 & 23092 & GTCACTCATAAGCGCGCTCAA \\
\hline CDK10 & CDK10-dplx1 & 8558 & CGCTATGTTGGAAATGTGCAA \\
\hline CDK10 & CDK10-dplx2 & 8558 & CAGGGACCTGAAGGTTTCCAA \\
\hline CDKL2 & CDKL2-dplx1 & 8999 & ATGATGTGTTTAGGTAATCTA \\
\hline CDKL5 & CDKL5-dplx1 & 6792 & AAGATAGACGCTTCATGTTAA \\
\hline DGKE & DGKE-dplx1 & 8526 & AACCCAAGCTTTGCGATTACA \\
\hline DGKE & DGKE-dplx2 & 8526 & CCCGCGCGAGGTATCGTCCTT \\
\hline DGKE & DGKE-dplx3 & 8526 & AAGGAAATGATTACTGTCAAA \\
\hline DGKE & DGKE-dplx4 & 8526 & ATCCATTAATCAGATGCGTAA \\
\hline DGKE & DGKE-dplx5 & 8526 & ACCCAAGGAATTCACAATGAA \\
\hline DGKE & DGKE-dplx6 & 8526 & ATCGATGGAAAGTTCAAGTAA \\
\hline DGKE & DGKE-dplx7 & 8526 & CTGGAAGTCGTTGGAGTATAT \\
\hline DGKE & DGKE-dplx8 & 8526 & CACCAAGTTATTTAACATCCA \\
\hline DGKE & DGKE-dplx9 & 8526 & ATGAATAGTATTAACTTACAA \\
\hline EPHA8 & EPHA8-dplx1 & 2046 & ACGGCCCACCAGGGTATGTAA \\
\hline MAP4K1 & MAP4K1-dplx1 & 11184 & CTGACTAAGAGTCCCAAGAAA \\
\hline MAP4K1 & MAP4K1-dplx2 & 11184 & TACGTGGGTGTACTCCATCAA \\
\hline MAPK11 & MAPK11-dplx1 & 5600 & CAGGATGGAGCTGATCCAGTA \\
\hline PAK7 & PAK7-dplx1 & 57144 & ATGATCTGGATCCGTATTATA \\
\hline PHKB & PHKB-dplx1 & 5257 & AGGGCTGTTCGTGGTCAGTTA \\
\hline Ryk & Ryk-dplx2 & 6259 & AAGGTGAAGGATATAGCAATA \\
\hline SAST & SAST-dplx1 & 22983 & CCGCGCTTTCTAATTTCTOGA \\
\hline SAST & SAST-dplx2 & 22983 & CACCTOCATGGGTCACATCAA \\
\hline SIK2 & SIK2-dplx2 & 23235 & CCGAAGGATGTTGGTCCTAGA \\
\hline TGFBR2 & TGFBR2-dplx1 & 7048 & CTCCAATATCCTCGTGAAGAA \\
\hline
\end{tabular}

